\title{
OPEN Graphene perfect absorber with loss adaptive $Q$-factor control function enabled by quasi-bound states in the continuum
}

\author{
Sangjun Lee, Joohyung Song \& Sangin Kim ${ }^{凶}$
}

Numerous device structures have been proposed for perfect absorption in monolayer graphene under single-sided illumination, all of which requires the critical coupling condition, i.e., the balance between the loss of graphene and the leakage rate of the device. However, due to the difficulty of the precise control of the quality of synthesized graphene and unwanted doping in graphene transferred to the substrate, the loss of graphene is rather unpredictable, so that the perfect absorption is quite difficult to achieve in practice. To solve this problem, we designed a novel perfect absorber structure with a loss adaptive leakage rate control function enabled by the quasi-bound states in the continuum (BIC) and numerically demonstrated its performance. Our designed device is based on a slab-waveguide grating supporting both the quasi-BIC and the guided-mode resonance (GMR); the quasi-BIC with an adjustable leakage rate controlled by an incident angle is responsible for absorption, while the GMR works as an internal mirror. Since the proposed device scheme can have an arbitrarily small leakage rate, it can be used to implement a perfect absorber for any kind of ultrathin absorbing media. Due to the simple structure avoiding an external reflector, the device is easy to fabricate.

Graphene has attracted strong interests in developing high-speed photodetectors due to its high carrier mobility ${ }^{1-4}$. Undoped monolayer graphene exhibits uniform light absorption efficiency of $\sim 2.3 \%$ over a wideband wavelength range from visible to terahertz. Considering its atomically ultrathin thickness of $\sim 0.34 \mathrm{~nm}$, the absorption efficiency is relatively high value. However, for practical high-performance photodetectors, the absorption should be greatly enhanced by applying various resonant structures such as gratings or photonic crystals $^{5-8}$, prism couplers ${ }^{9,10}$, and Fabry-Perot cavities ${ }^{11,12}$. From a standpoint of absorption efficiency enhancement, perfect absorption under single-sided illumination is an ultimate solution. Over the past decade, three types of perfect absorber schemes have been proposed: a single-mode/mirror scheme $e^{5-16}$, a dual-mode (or degenerate critical coupling) schem $\mathrm{e}^{17,18}$, and a triple-mode scheme ${ }^{19}$. The single-mode/mirror scheme requires a rather complicate fabrication process due to Bragg reflector or suffers from unwanted loss due to metal reflector. Although the dual-mode scheme avoids the use of mirror, it is prohibitively restrictive to simultaneously satisfy the frequency degeneracy and the critical coupling conditions of dual modes. The triple-mode scheme relieves design complexity compared to the dual-mode scheme but requires somewhat complicate fabrication process due to an added slab structure. Recently, our group proposed another mirror-less perfect absorber scheme based on an asymmetric single resonator such as a slab-waveguide grating (SWG) by adopting a one-port mimicking concept ${ }^{20}$. In this scheme, two indirectly coupled degenerate guided-mode resonance (GMR) modes are used to achieve a virtual one-port system, in which only one mode experiences loss and the other functions as an internal reflector in conjunction with the Fabry-Perot (F-P) like background scattering. This scheme does not require any structural symmetry, and its performance shows greatly enhanced fabrication error tolerance in comparison to the previously proposed dual-mode scheme of ref. ${ }^{17}$.

In the perfect absorber schemes, the critical coupling condition, i.e., balance between loss and leakage (or decay) rates is a common and essential requirement. The leakage rates of the relevant resonant modes in all the forementioned perfect absorber schemes are determined by structural parameters and thus, can hardly be adjusted after fabrication. On the other hand, the loss rate of graphene is somewhat unpredictable since the quality and the thickness of synthesized graphene is quite difficult to control precisely ${ }^{10}$. Besides, the substrate can cause unwanted doping of graphene, which also changes the loss rate. So, the critical coupling condition for 
(a)

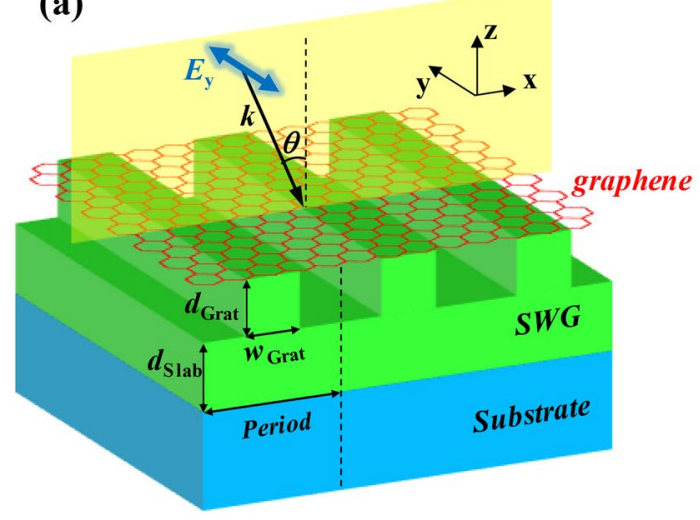

(b)

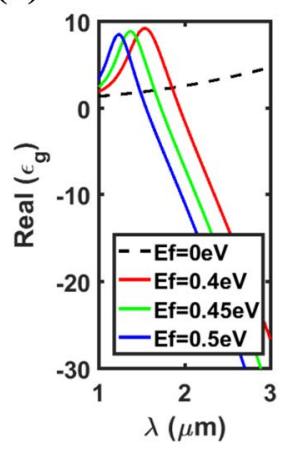

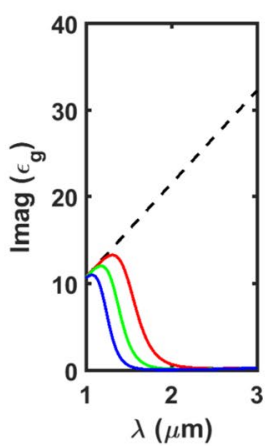

Figure 1. (a) Schematic of the proposed perfect absorber consisting of monolayer graphene placed on a slab-waveguide grating (SWG), where $n_{\mathrm{SWG}}=3.40, n_{\mathrm{Sub}}=1.45 . F F$ is defined as $w_{\text {Grat }} /$ Period. The red thin layer indicates monolayer graphene of $t_{G}=0.34 \mathrm{~nm}$ thickness as an absorbing medium. (b) Real and Imaginary part of permittivity $\left(\varepsilon_{\mathrm{g}}\right)$ of graphene for different Fermi-levels $\left(E_{\mathrm{f}}=0,0.4,0.45\right.$, and $\left.0.5 \mathrm{eV}\right)$ over the wavelength range of $\lambda=1-3 \mu \mathrm{m}$. The optimal $(A>99.99 \%)$ design parameters for $E_{\mathrm{f}}=0$ : Period $=0.85 \mu \mathrm{m}, d_{\text {Grat }}=0.367 \mu \mathrm{m}$, $d_{\text {Slab }}=0.052 \mu \mathrm{m}, F F=0.55$, and $\theta=3.72 \mathrm{deg}$ at $\lambda=1.5472 \mu \mathrm{m}$.

perfect absorption is quite difficult to satisfy in practice. This is the fundamental problem of all the previously proposed perfect absorber schemes. In this work, to solve this problem, we newly propose a perfect absorber scheme with a controllable leakage rate based on a quasi-bound states in the continuum (BIC).

The BIC is a special eigenstate that remains localized and has an infinite quality factor ( $Q$-factor) even though it exists within the light cone of the surrounding medium ${ }^{18,21-36}$. In practice, the BIC can be realized as a quasi-BIC whose finite $Q$-factor is adjusted by breaking structural symmetries ${ }^{18,24-32}$ or through parameter tuning ${ }^{33-36}$. Recently, quasi-BICs in various periodic structures (such as gratings or photonic crystals ${ }^{23-28,33-35}$, and metasurfaces ${ }^{18,29-32}$ ) or an isolated nanoparticle ${ }^{36}$ have attracted substantial attention as a platform for enhancement of light-matter interaction due to their ultrahigh $Q$-factor.

An interesting feature of the quasi-BICs in the periodic structures formed by breaking structural symmetries with oblique incidence of light is that their $Q$-factors are dependent on the incident angle ${ }^{24-28}$. Based on the controllable $Q$-factor of the quasi-BIC, we devised the practical perfect absorber scheme in which its leakage rate is adapted to the loss rate by a proper choice of the incidence angle. The basic concept of our proposed perfect absorber is the same as the asymmetric resonator-based one-port mimicking scheme ${ }^{20}$. Here, the quasi-BIC is adopted in the SWG as the lossy resonant mode and its resonance should be tuned to the GMR and the background scattering which forms an internal reflector function. In addition to the adaptive leakage rate, another outstanding feature of the proposed perfect absorber is considerably simple fabrication since absorbing medium (graphene) is placed on the ridge side of the SWG. This is because the quasi-BIC is mostly confined in the ridge region. In the designed device, for small value of asymmetry parameters, which is determined by an incidence angle, quadratic dependence of the $Q$-factor on the asymmetry parameter is confirmed. To investigate the reflection and absorption spectra, and field profiles of various resonant modes in the proposed absorber, we used the rigorous coupled wave analysis (RCWA) method ${ }^{37}$. The incidence angle dependence of quasi-BIC is also analyzed by using the coupled mode theory $(\mathrm{CMT})^{5,6,38}$.

\section{Results \& discussion}

The proposed absorber (Fig. 1a) consists of a one-dimensional (1-D) SWG of Si stacked on a low-index substrate $\left(\mathrm{SiO}_{2}\right)$, where the ridge (grating) and the sublayer (slab) possess the same index, and monolayer graphene placed just above the SWG. Refractive indices of $\mathrm{Si}$ and $\mathrm{SiO}_{2}$ are 3.40 and 1.45, respectively. For the permittivity of graphene, Kubo formulation ${ }^{19,39}$ was used with parameters of a graphene thickness of $0.34 \mathrm{~nm}$, Fermi velocity of $10^{6} \mathrm{~m} / \mathrm{s}$, and mobility of $0.5 \mathrm{~m}^{2} / \mathrm{Vs}$. Figure $1 \mathrm{~b}$ shows real and imaginary parts of the permittivity $\left(\varepsilon_{\mathrm{g}}\right)$ of graphene for different Fermi-levels $\left(E_{\mathrm{f}}=0,0.4,0.45\right.$, and $\left.0.5 \mathrm{eV}\right)$ over the wavelength range of $\lambda=1 \sim 3 \mu \mathrm{m}$. We consider a transverse electric (TE, or $s$-polarized) wave obliquely incident from the air with an incidence angle of $\theta$, whose electric field is perpendicular to the incidence plane. The fill factor $(F F)$ is defined as $w_{\text {Grat }} /$ Period. The optimal design parameters are as follows: Period $=0.85 \mu \mathrm{m}, d_{\text {Grat }}=0.367 \mu \mathrm{m}, d_{\text {Slab }}=0.052 \mu \mathrm{m}, F F=0.55$, and $\theta=3.72 \mathrm{deg}$ at $\lambda=1.5472 \mu \mathrm{m}$, which are designed to obtain perfect absorption $(A>99.99 \%)$ in undoped $\left(E_{\mathrm{f}}=0\right)$ monolayer graphene at $\lambda \sim 1.55 \mu \mathrm{m}$.

The performance of the designed graphene perfect absorber is numerically demonstrated, especially focused on the loss variation adaptive $Q$-factor control via a proper choice of the incidence angle. All numerical calculation in this work has been conducted with the RCWA simulation ${ }^{37}$. Figure 2 a shows the calculated absorption spectra as a function of $\theta$ for the designed perfect absorber with undoped graphene $\left(E_{\mathrm{f}}=0\right)$. A strong absorption peak branch is observed for $\sim 2$ degree $<\theta<\sim 6$ degree, and perfect absorption $(A>99.995 \%)$ occurs at $\theta=$ 3.72 degree and $\lambda=1.5472 \mu \mathrm{m}$ as marked by the white open circle. The enhanced absorption is attributed to the quasi-BIC mode, which will be confirmed later in Fig. 5b. The absorption spectrum for the optimal angle $(\theta=$ 
(a)

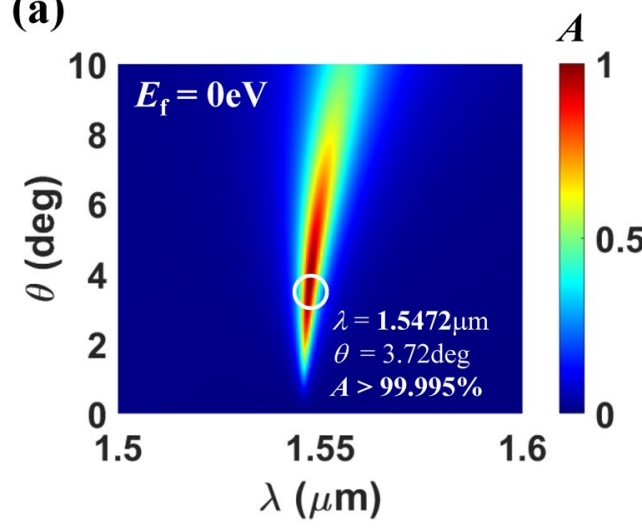

(c)

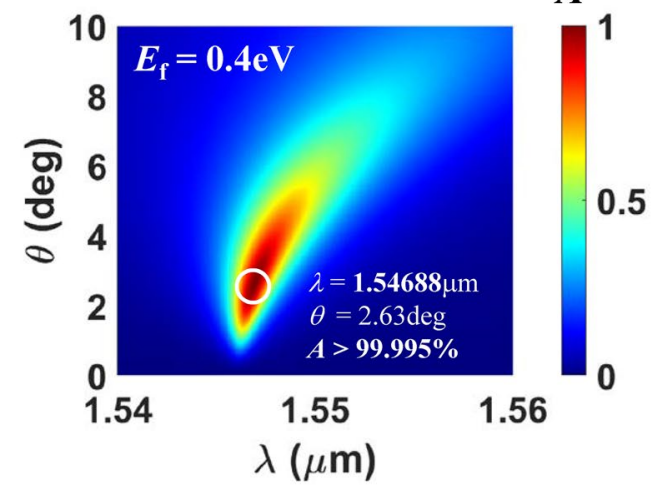

(b)

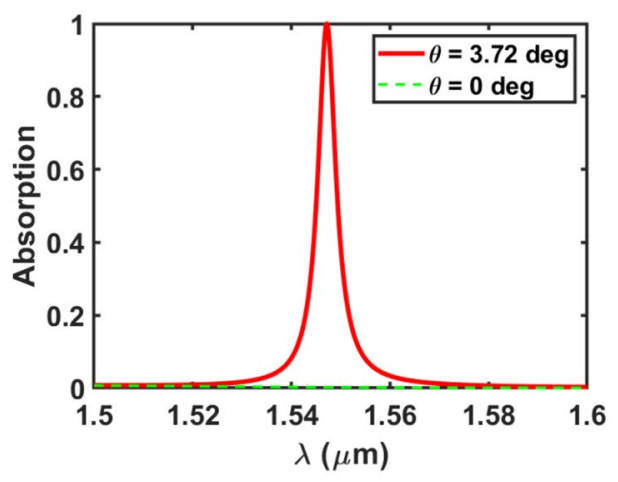

(d)

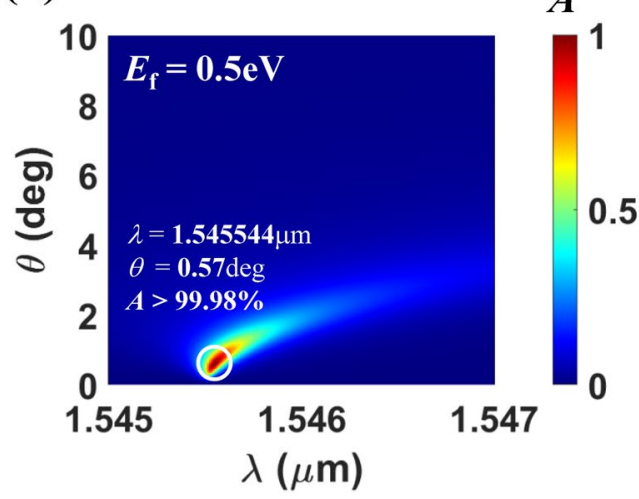

Figure 2. (a) Absorption spectra as a function of $\theta$ for the proposed graphene absorber, where $E_{\mathrm{f}}=0 \mathrm{eV}$, Period $=0.85 \mu \mathrm{m}, F F=0.55, d_{\text {Grat }}=0.367 \mu \mathrm{m}$, and $d_{\text {Slab }}=0.052 \mathrm{~m} \mu$. (b) Absorption spectra extracted at two incidence angles $(\theta=0$ and $3.72 \mathrm{deg})$, which are extracted from (a). Absorption spectra as a function of $\theta$ when (c) $E_{\mathrm{f}}=0.4 \mathrm{eV}$ and (d) $E_{\mathrm{f}}=0.5 \mathrm{eV}$, assuming that all the remaining parameters are same as those of $(\mathbf{a})$.

3.72 degree) is compared to that for the normal incidence case in Fig. 2b. For the normal incidence, only a GMR mode is excited since the excitation of the symmetry-protected (ideal) BIC mode is forbidden. So, the small absorption $(A<<1 \%)$ for the normal incidence implies that the absorption due to the GMR mode is negligible and the one-port system mimicking concept really works. The detailed analysis on the operation principle of the designed device will be discussed in the next section.

The excellent feature of the designed perfect absorber is that loss adaptive perfect absorption is achievable via a proper choice of the incidence angle. Especially, perfect absorption can be achieved even if the loss (or the absorption coefficient) of the absorbing material is extremely low. This is attributed to the unique property of the quasi-BIC with an extremely large $Q$-factor (a vanishingly small leakage rate) as the incident angle approaches zero. So, if the absorption coefficient of the absorbing material becomes smaller, the critical coupling condition, that is, the balance between the leakage rate and the loss rates can be satisfied at a smaller incident angle. To demonstrate this, we investigated the angle dependent absorption performance of the designed device for two different doping levels (or Fermi levels, i.e. $E_{\mathrm{f}}$ ) of graphene, which is plotted in Fig. $2 \mathrm{c}$,d. The loss of graphene rapidly decreases with a Fermi level increase as seen in Fig. 1b. For $E_{\mathrm{f}}=0.5 \mathrm{eV}$ and mobility $M o=0.5 \mathrm{~m}^{2} / \mathrm{Vs}$, the loss rate of graphene corresponds to $\sim 1 / 40$ of the undoped case of the same mobility at $\lambda \sim 1.55 \mu \mathrm{m}$. Despite of the significant loss rate change, perfect absorption is still achieved at a lower incidence angle. The bandwidth of absorption spectrum decreases as $E_{\mathrm{f}}$ increases, which is due to the increased Q-factor of the quasi-BIC mode at the lower incidence angle. It is straightforward that perfect absorption can also be achieved for the increased loss of graphene at the larger incidence angle, which is not shown here. Therefore, even for the unwanted loss variation of graphene, the designed device can achieve perfect absorption via adaptive control of the incidence angle.

Quasi-BICs in a SWG. In order to analyze the operation principle of the proposed perfect absorber, we first investigate the reflection properties when the absorbing medium is removed from the designed absorber. Figure 3a shows the fill factor $(F F)$ dependence of reflectance $\left(|R|^{2}\right)$ for normal incidence $(\theta=0)$, where only the GMR modes are excited. Note that no BIC mode is excited due to the lateral anti-symmetry. When $F F$ is very large or small, the reflectance branches become very narrow, corresponding to sharp spectral resonances, due to small leakage rates of the GMRs in the SWG that are noted as GMR ${ }^{1 s t}$ and $\mathrm{GMR}^{2 \mathrm{nd} 40,41}$. The field profiles of those modes at $F F=0.90$ are plotted in the upper panels of Fig. 3b, indicating their origins. In those plots, the field of the incident/reflected plane wave in the air is not clearly observable because of the strong field enhancement in the SWG caused by their high $Q$-factor. For moderate fill factors $(\sim 0.2<F F<\sim 0.6)$, the $Q$-factor of $\mathrm{GMR}^{1 \mathrm{st}}$ 
(a)

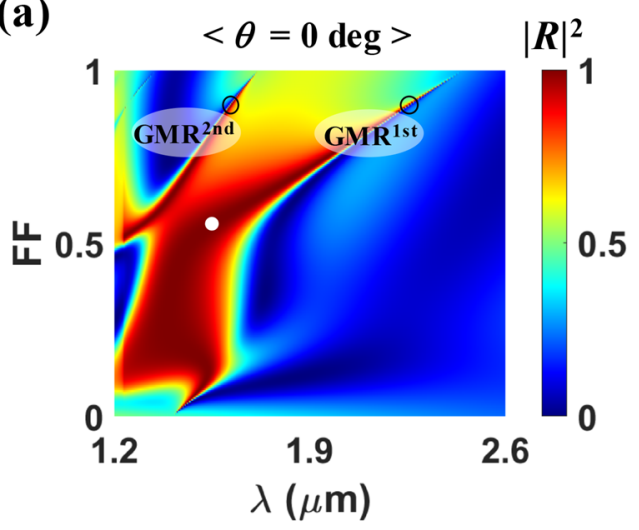

(b)
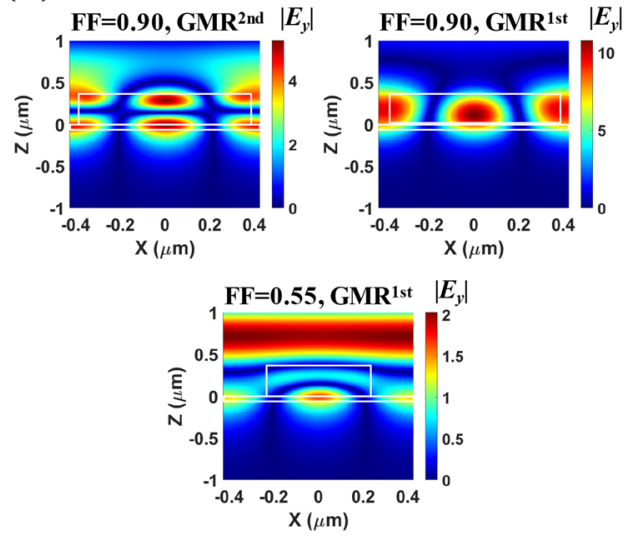

(c)

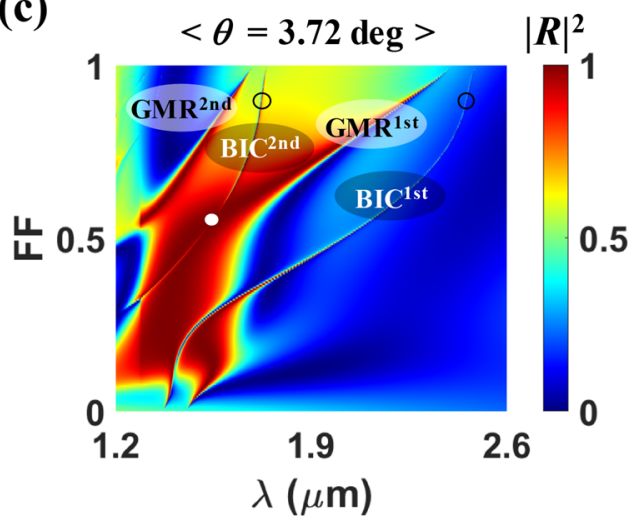

(d)
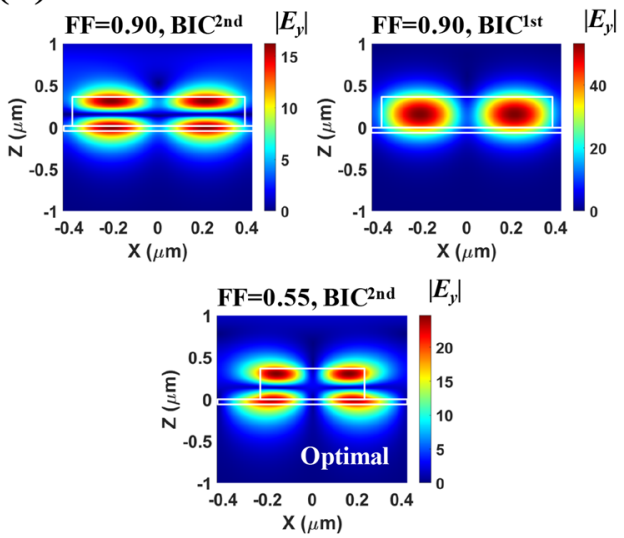

Figure 3. Reflectance spectra as a function of $F F$ at (a) $\theta=0$ (normal incidence) and (c) $\theta=3.72$ deg (oblique incidence) for the proposed structure without graphene, where Period $=0.85 \mu \mathrm{m}, d_{\text {Grat }}=0.367 \mu \mathrm{m}$, and $d_{\text {Slab }}=0.052 \mu \mathrm{m}$. (b) and (d) indicate the normalized electric field distributions $\left(\left|E_{y}\right|\right)$ at different conditions marked by open and solid circles in (a) and (c) respectively are plotted: $F F=0.90$ (upper panel), $F F=0.55$ (lower panel).

considerably decreases and thus, it forms a broadband reflector in conjunction with the F-P like background scattering ${ }^{20,42}$. As plotted in the lower panel of Fig. 3b, the field profile of the low-Q GMR ${ }^{1 s t}$ near the reflection peak condition $(F F=0.55, \lambda=1.5472 \mu \mathrm{m}, \theta=0$, which is marked by the white solid circle in Fig. 3a) shows relatively weak field amplitude in the SWG, which is significantly modified compared to the case of $F F=0.90$ owing to the strong scattering strength of the ridge part. However, the original guided-mode property is still observed. One important feature to note here is that most of the field is confined in the slab region of the SWG, so that $\mathrm{GMR}^{1 \mathrm{st}}$ mode will experience almost no loss when monolayer graphene is placed on top of the SWG.

For oblique incidence $(\theta=3.72$ degree $)$, two high-Q branches additionally appear on the reflectance map (Fig. 3c). They correspond to the first- and the second-order quasi-BIC modes noted as BIC ${ }^{1 s t}$ and BIC ${ }^{2 n d}$ and cross different regions of the broad reflection branch due to the GMR mode. These quasi-BIC modes are manifested by breaking the symmetry of 'symmetry-protected BICs' with infinite Q-factor ${ }^{24-28}$. Note that for the symmetry-protected BICs, leakage radiation to the surface normal direction is forbidden because of symmetry incompatibility with the external radiation, as checked in Fig. 3a. The symmetry-protection of the BIC originates from the destructive interaction of two leaky guided mode propagating in the opposite direction. So, for the oblique incidence, the imbalanced excitation of the two destructive leaky guided modes forms the imperfect (quasi) BIC which can be coupled to the external plane wave. The field profiles of $\mathrm{BIC}^{1 \text { st }}$ and $\mathrm{BIC}^{2 \mathrm{nd}}$ at $F F=0.90$ are plotted in the upper panels of Fig. $3 \mathrm{~d}$, where the vertical field distributions confirm that the quasi-BICs originates from the leaky guided modes. The quasi-BIC mode relevant to perfect absorption in our designed device is BIC $^{2 n d}$, which will be discussed later. As shown in the lower panel of Fig. $3 \mathrm{~d}$, at the optimal condition $(F F=0.55$, $\lambda=1.5472 \mu \mathrm{m}, \theta=3.72$ degree, which is marked by the white solid circle in Fig. $3 \mathrm{c}$ ), the electric field profile is not perfectly but close to anti-symmetric in the lateral $(x)$ direction with respect to the center of the ridge since the contribution of $\mathrm{BIC}^{2 \mathrm{nd}}$ is dominant. Although $\mathrm{GMR}^{1 \mathrm{st}}$ mode is also excited in this case, the energy stored in it is negligible compared to $\mathrm{BIC}^{2 \text { nd }}$ due to the huge $\mathrm{Q}$-factor difference. So, much stronger field enhancement is attributed to $\mathrm{BIC}^{2 \mathrm{nd}}$ as well.

Virtual one-port feature in the interaction between the GMR and the quasi-BIC modes and its angle dependence. Now, we closely investigate the interaction between the GMR and the quasi-BIC 
(a)

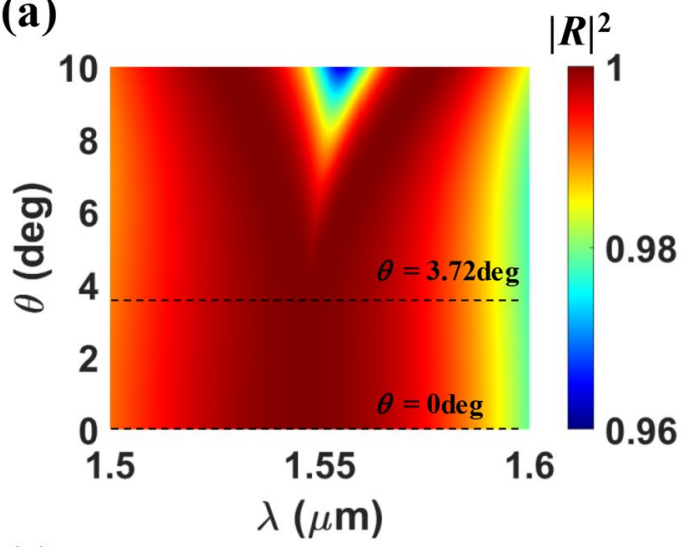

(c)

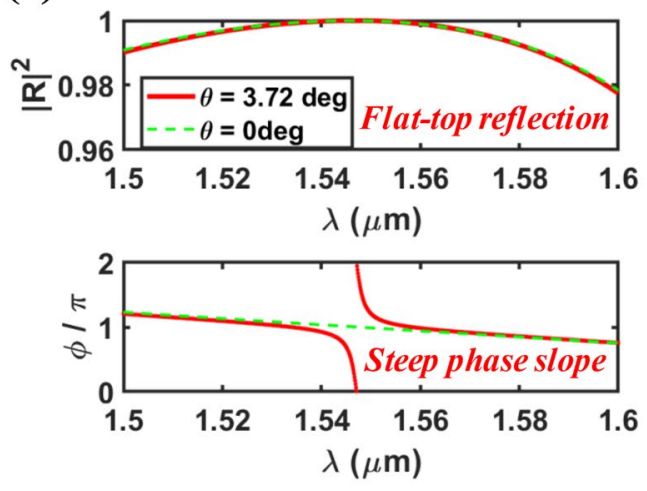

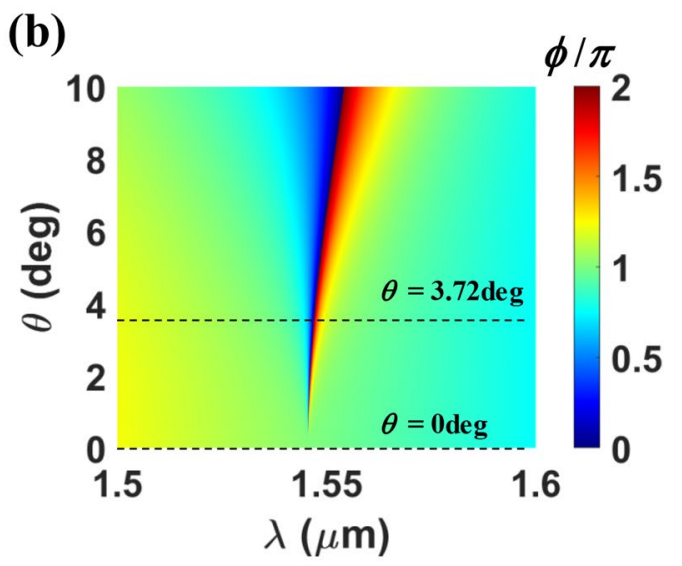

(d)

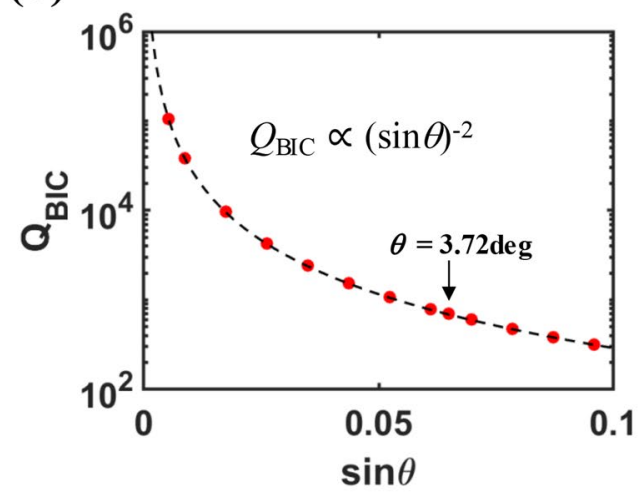

Figure 4. (a) Reflectance spectra and (b) reflection phase a function of $\theta$ for the proposed structure without graphene, assuming that all the remaining parameters are same as the optimal condition in Fig. 2 (that is, Period $=0.85 \mu \mathrm{m}, F F=0.55, d_{\text {Grat }}=0.367 \mu \mathrm{m}$, and $\left.d_{\text {Slab }}=0.052 \mu \mathrm{m}\right)$. (c) Reflectance spectra and reflection phase at two angles $(\theta=0$ and $3.72 \mathrm{deg}$ ), which are extracted from (a) and (b) respectively. (d) $Q$-factor of quasi-BIC $\left(Q_{\mathrm{BIC}}\right)$ as a function of $\theta$, which is extracted by applying CMT model: red solid circle is for CMT model, and black dashed curve is for a proper inverse quadratic equation $\left(Q_{\mathrm{BIC}}=2.9 \cdot(\sin \theta)^{-2}\right)$ as approximate expression.

modes, and the angle dependence of $\mathrm{BIC}^{2 \text { nd }}$ for the designed structure without graphene layer. Figure $4 \mathrm{a}, \mathrm{b}$ show reflectance $\left(|R|^{2}\right)$ and reflection phase $(\phi)$ spectra as a function of the incidence angle over the wavelength range of the high reflection due to $\mathrm{GMR}^{1 \mathrm{st}}$ mode at normal incidence $(\sim 1.5 \mu \mathrm{m}<\lambda<\sim 1.6 \mu \mathrm{m})$. The broadband flattop reflection close to $100 \%$ is maintained up to $\theta \sim 5$ degree, and as $\theta$ increases further, a reflection dip develops, reaching $|R|^{2} \sim 96 \%$ for $\theta=10$ degree. On the other hand, the phase shows steep variation near the wavelength of $\lambda \sim 1.55 \mu \mathrm{m}$ for $\theta>0$ and the wavelength of $\phi=0$ shows slow red shift with increasing $\theta$. From the fact that no steep phase variation is observed at normal incidence $(\theta=0)$, in which the excitation of the BIC is forbidden, we can see that the steep phase variation is due to the resonant excitation of the quasi-BIC (BIC ${ }^{\text {2nd }}$ ), and the wavelength of $\phi=0$ corresponds to the resonance wavelength of the quasi-BIC ( $\left.\mathrm{BIC}^{2 \mathrm{nd}}\right)$. We can see that the locus of the resonance wavelength of $\mathrm{BIC}^{2 \mathrm{nd}}$ is the same as the strong absorption peak branch in Fig. 2a, which is a clear evidence that $\mathrm{BIC}^{2 \mathrm{nd}}$ is responsible for the absorption in our designed device.

For a clear comparison, two reflectance (reflection phase) spectra for $\theta=0$ and 3.72 degree are plotted together in the upper (lower) panel in Fig. 4c. This reveals that in our designed device, the introduction of the high- $Q$ $\mathrm{BIC}^{2 \mathrm{nd}}$ does not ruin the broadband flat-top reflection due to the $\mathrm{GMR}^{1 \text { st }}$ - the background scattering interaction. It is noteworthy that this kind of interaction between two resonant modes in a single resonator is an unusual phenomenon, which requires triple degeneracy among the two resonant modes and the F-P like background scattering and a proper indirect mutual coupling strength between the two modes as theoretically proved in our previous works ${ }^{20,43}$. Unless these conditions are satisfied, which is more usual, a sharp reflection fluctuation known as Fano line shape appears ${ }^{44}$. Previously, quasi-BIC related Fano resonances are utilized for a narrowband transmission filter at slightly off-normal incidence angle in a 1-D high contrast grating (HGC) ${ }^{28}$ and a narrowband reflection filter in an asymmetric metasurface ${ }^{29}$. As seen in Fig. 3c, even in our designed structure, a sharp reflection dip of $R \sim 0$ is observed when BIC ${ }^{1 s t}$ interacts with the GMR mode at $F F=0.2$ and $\lambda=1.4465 \mu$ m with all other parameters kept the same as the optimal design. The enlarged spectrum plot at $F F=0.2$ (Fig. S1) and its detailed description is provided in Supplementary information. The interaction between two resonant modes in a two-port asymmetric resonator like the SWG can be modeled as an indirect coupling via the partial reflections at the two port interfaces in the $\mathrm{CMT}^{20,43}$. From this CMT modeling, we can find the specific indirect coupling condition between the two degenerate resonant modes for perfect reflection or transmission at the resonance. 
(Supplementary information) This implies that depending on the indirect mutual coupling condition between the quasi-BIC and the GMR modes, perfect transmission as well as perfect reflection can occur at the resonance. Since the indirect coupling stems from the partial reflections at the surfaces of the SWG, the coupling condition is strongly dependent on $F F$. In our optimal design, the proper coupling condition between BIC ${ }^{2 n d}$ and $\mathrm{GMR}^{1 s t}$ modes for perfect reflection is satisfied at $F F=0.55, \lambda=1.5472 \mu \mathrm{m}$, and $\theta=3.72$ degree, while perfect transmission condition happen to be satisfied between $\mathrm{BIC}^{1 \text { st }}$ and $\mathrm{GMR}^{1 \mathrm{st}}$ modes at $F F=0.2$ and $\lambda=1.4465 \mu \mathrm{m}$. Since the one-port resonant system mimicking concept requires perfect reflection at resonance, perfect absorption in our designed device is based on $\mathrm{BIC}^{2 \text { nd }}$ and $\mathrm{GMR}^{1 \text { st }}$ modes.

In our present design, the proper coupling condition between $\mathrm{BIC}^{2 \mathrm{nd}}$ and $\mathrm{GMR}^{1 \text { st }}$ modes appears to remain roughly up to $\theta \sim 5$ degree as seen in Fig. 4a. For a larger angle, the proper coupling condition becomes to break due to the unacceptably reduced $Q$-factor of $\mathrm{BIC}^{2 \text { nd }}$ mode, resulting in the reflectance dip development. Note that the indirect coupling strength is determined by the decay rates of the two modes as well as the reflections at the surfaces of the $\mathrm{SWG}^{20,43}$. So, the operation range of $\theta$ for the variable $Q$-factor should be limited. However, this is not the fundamental limit. Our present design is for undoped graphene of a certain condition (Fermi velocity of $10^{6} \mathrm{~m} / \mathrm{s}$, and mobility of $0.5 \mathrm{~m}^{2} / \mathrm{Vs}$ ). For graphene of a larger loss due to the difference material quality, the device can be redesigned to have a larger optimal incidence angle, and then, the operation range of $\theta$ can be increased.

It is also noteworthy that the reflection characteristics of our designed structure without graphene is similar to the phase shifter based on an ideal asymmetric Fabry-Perot cavity with a perfectly reflecting bottom mirror ${ }^{38,45,46}$. Therefore, it can be stated that the designed structure behaves like a virtual one-port resonant system, mimicking the system composed of a single-mode resonator and an external mirror. In our proposed perfect absorber structure, BIC $^{2 \text { nd }}$ works as a lossy mode while $\mathrm{GMR}^{1 \mathrm{st}}$ works as an internal mirror in conjunction with the background scattering as aforementioned.

Another key feature of the proposed structure is that the slope of the reflection phase at the resonance, which is directly related to $Q$-factor of $B^{2} C^{2 n d}$, shows $\theta$ dependence as seen in Fig. 4b. The steeper phase slope implies the higher $Q$-factor (or lower leakage rate); the smaller incidence angle, the higher $Q$-factor. This is because a smaller incidence angle induces a weaker structural asymmetry and consequently, a less distortion of the symmetryprotected BIC. For the quantitative analysis of the angle dependent $Q$-factor of $B^{2}{ }^{2 n d}$, we extracted the leakage rate of $\mathrm{BIC}^{2 \text { nd }}$ by fitting the CMT model to the reflection phase spectrum calculated with the RCWA. According to the CMT, the reflection phase in a lossless one-port system with a single resonance is given by

$$
\phi(\omega)=2 \tan ^{-1}\left(\frac{\omega-\omega_{o}}{\gamma_{l e a k}}\right),
$$

where $\omega_{0}$ and $\gamma_{\text {leak }}$ are a resonant frequency and a leakage (or external decay) rate, respectively,38. The $Q$-factor of $\mathrm{BIC}^{2 \mathrm{nd}}$ is calculated by $Q_{\mathrm{BIC}}=\omega_{\mathrm{o}} / 2 \gamma_{\text {leak }}$, which is plotted in Fig. $4 \mathrm{~d}$. In particular, $Q_{\mathrm{BIC}} \sim 702$ at the optimal angle of $\theta=3.72$ degree. As expected, $Q_{\mathrm{BIC}}$ increases toward infinity as $\theta$ approaches zero. For a small incident angle, $Q_{\mathrm{BIC}}$ is inversely proportional to the square of the asymmetry parameter $(\sin \theta)$, similar to the result reported in ref. $^{29}$ in which asymmetry was introduced by the rotation angle of the individual meta-atom in the asymmetric metasurface for a narrowband reflection filter.

Optimal incidence angle selection for loss adaptive perfect absorption. The optimal incidence angle selection for perfect absorption satisfying the critical coupling condition is analyzed with the CMT. According to the CMT, absorption efficiency in a lossy one-port system with a single resonance is given by

$$
A(\omega)=\frac{4 \gamma_{l e a k} \gamma_{\text {loss }}}{\left(\omega-\omega_{o}\right)^{2}+\left(\gamma_{\text {leak }}+\gamma_{\text {loss }}\right)^{2}},
$$

where $\gamma_{\text {leak }}$ and $\gamma_{\text {loss }}$ and are the leakage and the loss (or the internal decay) rates, respectively ${ }^{5,6}$. Perfect absorption can be reached when the critical coupling condition $\left(\gamma_{\text {leak }}=\gamma_{\text {loss }}\right)$ is satisfied at the resonance $\left(\omega=\omega_{0}\right)$.

Once $\gamma_{\text {leak }}$ is obtained via fitting the CMT model (1) to the reflection phase spectrum calculated with the RCWA as mentioned in the previous section, we can also extract $\gamma_{\text {loss }}$ by fitting the CMT model (2) to the absorption spectra (Fig. 2) calculated with the RCWA, which are plotted as a function of $\theta$ in Fig. 5a. As $\theta$ increases, $\gamma_{\text {leak }}$ increases rapidly, while $\gamma_{\text {loss }}$ is almost constant, which is consistent with the physical reasoning. From this, it is straightforward that the optimal incidence angle will be changed if the loss rate of the absorbing material varies. In our designed absorber with undoped graphene $\left(E_{\mathrm{f}}=0\right)$, it is confirmed that the critical coupling occurs at $\theta=3.72$ degree, which is the optimal incidence angle for perfect absorption. For the doped graphene cases of $E_{\mathrm{f}}=0.4 \mathrm{eV}$ and $0.5 \mathrm{eV}$, the optimal incidence angles for the critical couplings are $\theta=2.63$ degree and 0.57 degree, respectively. In Fig. 5b, the numerically calculated absorption spectra and the CMT model are compared for the designed absorber with undoped graphene at the optimal incidence angle, where we can see the excellent agreement between them. This confirms again that the designed absorber behaves like a lossy one-port resonant system in the vicinity of the $\mathrm{BIC}^{2 \text { nd }}$ resonance.

\section{Conclusion}

In conclusion, we have numerically demonstrated a graphene perfect absorber with a function of adaptive control of $Q$-factor for loss variation, which is based on the quasi-BIC and the GMR modes in a SWG. In the device design, the one-port resonant system mimicking concept was adopted. The device structure is quite simple and easy to fabricate; no external mirror is required, and monolayer graphene is placed on the ridge of the SWG. The proposed perfect absorber scheme can be used for any kind of ultrathin absorbing media including various 2-D materials. 
(a)

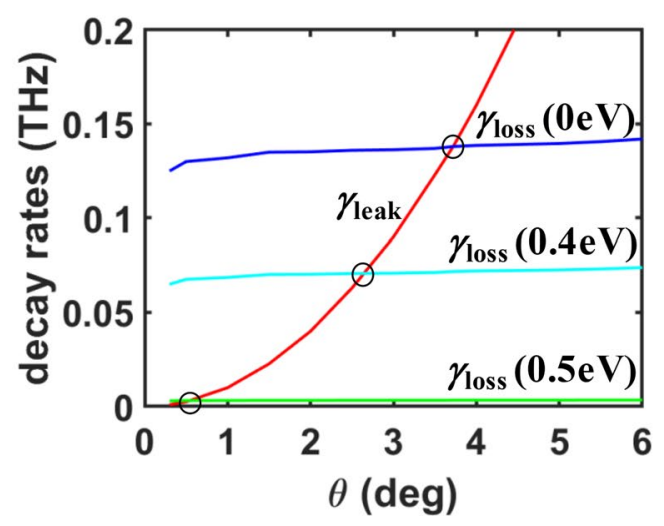

(b)

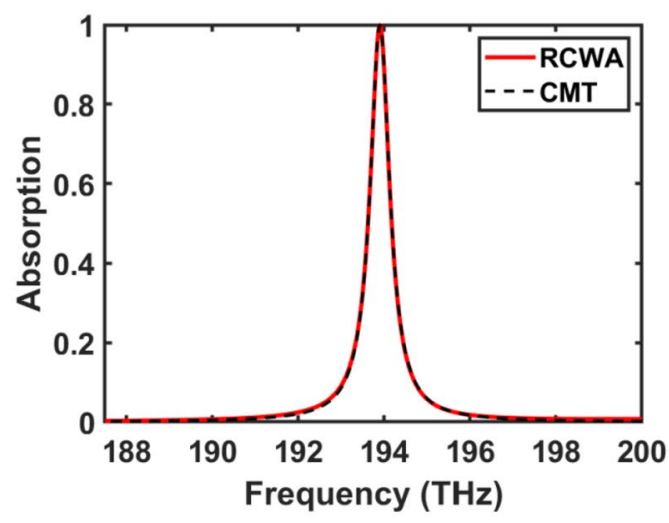

Figure 5. (a) Leakage and loss rates as a function of $\theta$ for different Fermi-levels of $E_{\mathrm{f}}=0,0.4$, and $0.5 \mathrm{eV}$, which are obtained by applying CMT model. Three open circles indicate the critical coupling condition for each $E_{\mathrm{f}}$. (b) Comparison of CMT and the RCWA calculation results for $E_{\mathrm{f}}=0 \mathrm{eV}$. All the remaining parameters are same as those of Fig. 2.

\section{Methods}

To numerically investigate and analyze the reflection and absorption properties in the proposed perfect absorber, we used two-dimensional RCWA (a commercial software, DiffractMOD) ${ }^{37}$ and the coupled mode theory (CMT) fitting for a lossy and lossless one-port resonant system ${ }^{5,6,38}$. In the RCWA simulation, more than 300 harmonics were applied to guarantee accuracy around the resonant wavelength. In all our calculations, the complex permittivity of graphene $\left(\varepsilon_{g}\right)$ was calculated using Kubo formulation based on the local random phase approximation for various $E_{\mathrm{f}}^{19,39}$, assuming graphene thickness of $0.34 \mathrm{~nm}$, Fermi velocity of $10^{6} \mathrm{~m} / \mathrm{s}$, and mobility of $0.5 \mathrm{~m}^{2} / \mathrm{Vs}$.

Received: 1 September 2021; Accepted: 15 November 2021

Published online: 24 November 2021

\section{References}

1. Liu, C.-H., Chang, Y.-C., Norris, T. B. \& Zhong, Z. Graphene photodetectors with ultra-broadband and high responsivity at room temperature. Nat. Nanotechnol. 9, 273-278 (2014).

2. Zhang, Y. et al. Broadband high photoresponse from pure monolayer graphene photodetector. Nat. Commun. 4, 1811 (2013).

3. Gan, X. et al. Chip-integrated ultrafast graphene photodetector with high responsivity. Nat. Photon. 7, 883-887 (2013).

4. Mueller, T., Xia, F. \& Avouris, P. Graphene photodetectors for high-speed optical communications. Nat. Photon. 4, 297-301 (2010).

5. Piper, J. R. \& Fan, S. Total absorption in a graphene monolayer in the optical regime by critical coupling with a photonic crystal guided resonance. ACS Photon. 1, 347-353 (2014).

6. Lee, S., Heo, H. \& Kim, S. High fabrication-tolerant narrowband perfect graphene absorber based on guided-mode resonance in distributed Bragg reflector. Sci. Rep. 9, 4294 (2019).

7. Long, Y., Shen, L., Xu, H., Deng, H. \& Li, Y. Achieving ultranarrow graphene perfect absorbers by exciting guided-mode resonance of one-dimensional photonic crystals. Sci. Rep. 6, 32312 (2016)

8. Guo, C.-C. et al. Experimental demonstration of total absorption over $99 \%$ in the near infrared for monolayer-graphene-based subwavelength structures. Adv. Opt. Mater. 4, 1955-1960 (2016).

9. Lee, S., Heo, H. \& Kim, S. Graphene perfect absorber of ultra-wide bandwidth based on wavelength-insensitive phase matching in prism coupling. Sci. Rep. 9, 11967 (2019).

10. Pirruccio, G., Moreno, L. M., Lozano, G. \& Rivas, J. G. Coherent and broadband enhanced optical absorption in graphene. ACS Nano 7, 4810-4817 (2013).

11. Furchi, M. et al. Microcavity-integrated graphene photodetector. Nano Lett. 12, 2773-2777 (2012).

12. Engel, M. et al. Light-matter interaction in a microcavity-controlled graphene transistor. Nat. Commun. 3, 906 (2012).

13. Zhu, L. et al. Angle-selective perfect absorption with two-dimensional materials. Light. Sci. Appl. 5, 1 (2016).

14. Yao, Y. et al. Electrically tunable metasurface perfect absorbers for ultrathin mid-infrared optical modulator. Nano. Lett. 14, 6526-6532 (2014).

15. Kim, S. et al. Electronically Tunable Perfect Absorption in Graphene. Nano. Lett. 18, 971-979 (2018).

16. Chen, X. et al. Electrically Tunable Perfect Terahertz Absorber Based on a Graphene Salisbury Screen Hybrid Metasurface. Adv. Optical Mater. 8, 1900660 (2020).

17. Piper, J. R., Liu, V. \& Fan, S. Total absorption by degenerate critical coupling. Appl. Phys. Lett. 104, 251110 (2014).

18. Tian, J. et al. High-Q All-Dielectric Metasurface: Super and Suppressed Optical Absorption. ACS Photon. 7, 1436-1443 (2020).

19. Lee, S., Tran, T. Q., Heo, H., Kim, M. \& Kim, S. A proposal of a perfect absorber with enhanced design and fabrication tolerance. Sci. Rep. 7, 4760 (2017)

20. Lee, S., Song, J. \& Kim, S. Graphene perfect absorber design based on an approach of mimicking one-port system in an asymmetric single resonator. Opt. Express 29, 29631-29640 (2021).

21. Koshelev, K., Favraud, G., Bogdanov, A., Kivshar, Y. \& Fratalocchi, A. Nonradiating photonics with resonant dielectric nanostructures. Nanophotonics 8, 725-745 (2019).

22. Koshelev, K., Bogdanov, A. \& Kivshar, Y. Meta-optics and bound states in the continuum. Sci. Bull. 64, 836-842 (2019).

23. Hsu, C. W. et al. Observation of trapped light within the radiation continuum. Nature 499, 188-191 (2013). 
24. Lee, S.-G. \& Magnusson, R. Band flips and bound-state transitions in leaky-mode photonic lattices. Phys. Rev. B 99, 045304 (2019).

25. Krasikov, S. D., Bogdanov, A. A. \& Iorsh, I. V. Nonlinear bound states in the continuum of a one-dimensional photonic crystal slab. Phys. Rev. B 97, 224309 (2018).

26. Fan, K., Shadrivov, I. V. \& Padilla, W. J. Dynamic bound states in the continuum. Optica 6, 169-173 (2019).

27. Yoon, J. W., Song, S. H. \& Magnusson, R. Critical field enhancement of asymptotic optical bound states in the continuum. Sci. Rep. 5, $18301(2015)$.

28. Foley, J. M., Young, S. M. \& Phillips, J. D. Narrowband mid-infrared transmission filtering of a single layer dielectric grating. Appl. Phys. Lett. 103, 071107 (2013).

29. Koshelev, K., Lepeshov, S., Liu, M., Bogdanov, A. \& Kivshar, Y. Asymmetric Metasurfaces with High-Q Resonances Governed by Bound States in the Continuum. Phys. Rev. Lett. 121, 193903 (2018).

30. Liu, M. \& Choi, D.-Y. Extreme Huygens' Metasurfaces Based on Quasi-Bound States in the Continuum. Nano Lett. 18, 8062-8069 (2018).

31. Campione, S. et al. Broken Symmetry Dielectric Resonators for High Quality Factor Fano Metasurfaces. ACS Photonics 3, 23622367 (2016).

32. Tittl, A. et al. Imaging-based molecular barcoding with pixelated dielectric metasurfaces. Science 360, 1105-1109 (2018).

33. Marinica, D. C., Borisov, A. G. \& Shabanov, S. V. Bound States in the Continuum in Photonics. Phys. Rev. Lett. 100, 183902 (2008).

34. Yang, Y., Peng, C., Liang, Y., Li, Z. \& Noda, S. Analytical Perspective for Bound States in the Continuum in Photonic Crystal Slabs. Phys. Rev. Lett. 113, 037401 (2014).

35. Hsu, C. W. et al. Bloch surface eigenstates within the radiation continuum. Light.: Sci. Appl. 2, e84 (2013).

36. Rybin, M. V. et al. High-Q supercavity modes in subwavelength dielectric resonator. Phys. Rev. Lett. 119, 243901 (2017).

37. Moharam, M. G., Grann, E. B., Pommet, D. A. \& Gaylord, T. K. Formulation for stable and efficient implementation of the rigorous coupled-wave analysis of binary gratings. J. Opt. Soc. Am. A 12, 1068-1076 (1995).

38. Qu, C. et al. Tailor the Functionalities of Metasurfaces Based on a Complete Phase Diagram. Phys. Rev. Lett. 115, 235503 (2015).

39. Koppens, F. H. L., Chang, D. E. \& García de Abajo, F. J. Graphene plasmonics: A platform for strong light matter interactions. Nano Lett. 11, 3370-3377 (2011).

40. Niraula, M., Yoon, J. W. \& Magnusson, R. Mode-coupling mechanisms of resonant transmission filters. Opt. Express 22, 2581725829 (2014).

41. Karagodsky, V. \& Chang-Hasnain, C. J. Physics of near-wavelength high contrast gratings. Opt. Express 20, 10888-10895 (2012).

42. Heo, H., Lee, S. \& Kim, S. Tailoring Fano resonance for flat-top broadband reflectors based on single guided-mode resonance. J. Lightwave Technology 37, 4244-4250 (2019).

43. Song, J., Heo, H., Lee, S. \& Kim, S. Mirror-less unidirectional radiation in an asymmetric single resonator. Preprint at https://doi. org/10.36227/techrxiv.15020034.v1 (2021).

44. Zhou, W. et al. Progress in 2D photonic crystal Fano resonance photonics. Prog. Quant. Electron. 38, 1-74 (2014).

45. Colburn, S., Zhan, A. \& Majumdar, A. Tunable metasurfaces via subwavelength phase shifters with uniform amplitude. Sci. Rep. 7, 40174 (2017).

46. Kondo, T., Ura, S. \& Magnusson, R. Design of guided-mode resonance mirrors for short laser cavities. J. Opt. Soc. Am. A 32, 1454-1458 (2015).

\section{Acknowledgements}

This work was supported by the National Research Foundation of Korea (2020R1A2B5B01002681, 2021R1A4A1033155).

\section{Author contributions}

S.L. proposed the perfect absorber scheme, S.L. and J.S. completed the numerical simulations and analytic mode development, and S.K. supervised the simulations and the analytical model development. All the authors discussed the results and contributed to the writing of the manuscript.

\section{Competing interests}

The authors declare no competing interests.

\section{Additional information}

Supplementary Information The online version contains supplementary material available at https://doi.org/ 10.1038/s41598-021-02318-8.

Correspondence and requests for materials should be addressed to S.K.

Reprints and permissions information is available at www.nature.com/reprints.

Publisher's note Springer Nature remains neutral with regard to jurisdictional claims in published maps and institutional affiliations.

(c) (1) Open Access This article is licensed under a Creative Commons Attribution 4.0 International License, which permits use, sharing, adaptation, distribution and reproduction in any medium or format, as long as you give appropriate credit to the original author(s) and the source, provide a link to the Creative Commons licence, and indicate if changes were made. The images or other third party material in this article are included in the article's Creative Commons licence, unless indicated otherwise in a credit line to the material. If material is not included in the article's Creative Commons licence and your intended use is not permitted by statutory regulation or exceeds the permitted use, you will need to obtain permission directly from the copyright holder. To view a copy of this licence, visit http://creativecommons.org/licenses/by/4.0/.

(C) The Author(s) 2021 